University of Wollongong

Research Online

Faculty of Engineering and Information

Faculty of Engineering and Information

Sciences - Papers: Part B

Sciences

2010

\title{
Humanoid human-like reaching control based on movement primitives
}

Minh Tuan Tran

Universite Paul Sabatier

Philippe Souères

LAAS-CNRS Toulouse France

Michel Taix

Universite Paul Sabatier

Manish Sreenivasa

University of Wollongong, manishs@uow.edu.au

Christophe Halgand

LAAS-CNRS Toulouse France

Follow this and additional works at: https://ro.uow.edu.au/eispapers1

Part of the Engineering Commons, and the Science and Technology Studies Commons

Research Online is the open access institutional repository for the University of Wollongong. For further information contact the UOW Library: research-pubs@uow.edu.au 


\title{
Humanoid human-like reaching control based on movement primitives
}

\author{
Abstract \\ This paper deals with the problem of generating realistic human-like reaching movements from a small \\ set of movement primitives. Two kinds of movement databases are used as reference. The first one is \\ obtained numerically, by applying biological principles of motor control on the dynamic model of the robot \\ arm. The second one is obtained by recording reaching movements of human subjects. From these \\ databases, primitives are extracted and analyzed by using Principal Component Analysis. An original \\ generalization method is then proposed for generating movements that did not belong to the initial \\ database. We show that twenty primitives allow to produce new movements, having characteristics \\ similar to that of humans. Experiments on the humanoid robot HRP-2 are presented to illustrate the result. \\ Keywords \\ primitives, humanoid, human-like, control, movement, reaching \\ Disciplines \\ Engineering I Science and Technology Studies

\section{Publication Details} \\ Tuan, T. M., Soueres, P., Taix, M., Sreenivasa, M. N. \& Halgand, C. (2010). Humanoid human-like reaching \\ control based on movement primitives. 19th International Symposium in Robot and Human Interactive \\ Communication (pp. 546-551). United States: IEEE.
}




\title{
Humanoid human-like reaching control based on movement primitives
}

\author{
Tran Minh Tuan, Philippe Souères, Michel Taïx, Manish N. Sreenivasa and Christophe Halgand
}

\begin{abstract}
This paper deals with the problem of generating realistic human-like reaching movements from a small set of movement primitives. Two kinds of movement databases are used as reference. The first one is obtained numerically, by applying biological principles of motor control on the dynamic model of the robot arm. The second one is obtained by recording reaching movements of human subjects. From these databases, primitives are extracted and analyzed by using Principal Component Analysis. An original generalization method is then proposed for generating movements that did not belong to the initial database. We show that twenty primitives allow to produce new movements, having characteristics similar to that of humans. Experiments on the humanoid robot HRP-2 are presented to illustrate the result.
\end{abstract}

\section{INTRODUCTION}

One of the main theories of motor control in biology conjectures that the Central Nervous System (CNS) of primates uses a finite set of elementary motor components, called primitives, to generate movements. This theory was suggested by numerous biological experiments. At the kinematic level, the primitives are sometimes described as joint covariations. For example, covariations of ankle, knee and hip joints during bending movements and walking were described in [1] and [2]. A similar coactivation between wholebody links during reaching movements was reported in [3] and [4]. Motor primitives or synergies were also pointed out at the muscular level, from EMG measurements. For instance, five muscular synergies involved in postural control were described in [5]. The encoding of motor primitives was also shown at the neural level. The well known experiment by Mussa-Ivaldi and Bizzi [6], showing that local stimulation of the spinal chord induce different leg movements in frogs, is a good illustration of this. Recently, an attempt at modeling these experimental results in the control theory framework was proposed in [7]. For roboticists, the idea of reducing the complexity of control by using a finite set of movement primitives is very attractive. It offers a promising alternative to the computation of inverse kinematics and cost minimization to cope with the high redundancy of anthropomorphic structures. This idea has already motivated some applications. Two primitives were used by Hauser et al. [8], for controlling the balance of a small size humanoid robot. Lim et al. [9], used Principal Component Analysis (PCA) to extract primitives from the captured movement of a human arm modeled as 4 Degrees Of Freedom (DOF) chain. These primitives were used as basis functions for parameterizing new realistic robot movements. In the study of Chhabra et. al. [10], the authors used a nonnegative matrix factorization method to extract primitives from a database of control signals to control a 2 DOF arm. Interestingly, it was shown that new movements can be learned faster in
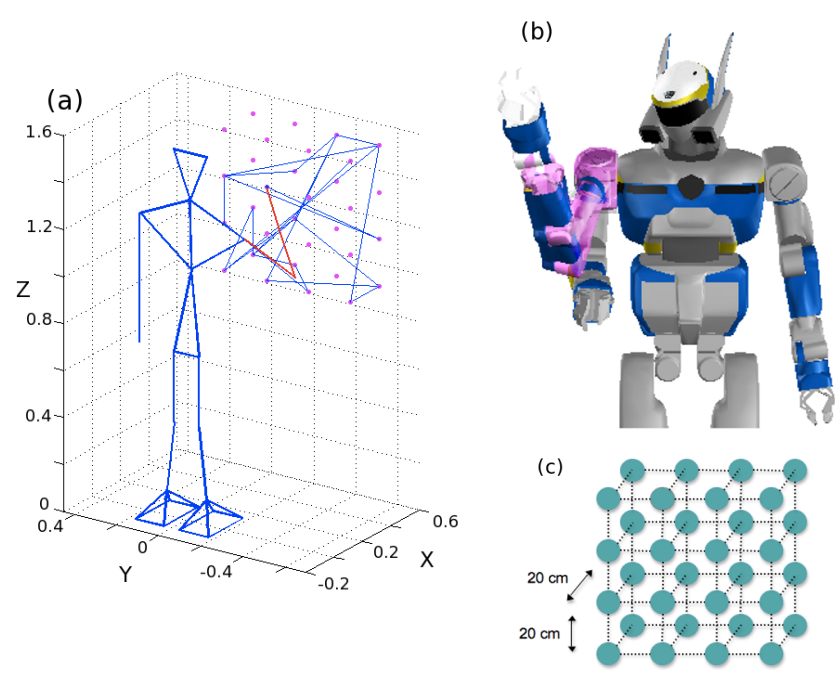

Fig. 1. (a) Illustration of the human experiment during successive reaching movements. The arm segments are in red and examples of hand trajectories are drawn in blue (b) The humanoid robot HRP-2 (Kawada Industries Inc., Japan). (c) Representation of the 32 target positions on two parallel virtual grids. These target positions were used to drive the human reaching movements

the primitive space than in the control space [11]. In order to introduce feedback in the control, Todorov et al. [12] considered sensorimotor primitives. This kind of primitive was applied to the control a 2 DOF arm.

In this paper, we propose a method that allow to characterize a set of kinematic primitives from a database of movements and use them to produce realistic human-like movements with a 6 DOF humanoid robot arm (Fig. 1-b). The movements are expected to have the main kinematic characteristics of human movements, namely: almost straight hand trajectories with a possible weak regular curvature, and bell-shaped velocity profiles. In order to develop a sufficiently generic method that allow an easy transfer from the human movement to the robot motion, we based our study on two different databases. The first database was obtained by simulation by applying a biologiocal model of motor control on the dynamic model of HRP-2's arm [13], [14]. The second database was constructed by recording human reaching movement on different subjects using a motion capture system. From both databases, primitives are extracted by using PCA, and the reconstruction process, which allows to express each movement as a linear combination of these primitives, is analyzed. Then, an original generalization method is proposed to generate new movements from this basis of primitives. The proposed method consists in solving a low-dimensional minimization problem to determine the 
weighting coefficients that allow to approach the minimumjerk solution with the best precision. Depending on the number of primitives, the computation-time and the precision of the reconstruction processes, are discussed. Finally, examples of reaching movements computed by this method are presented on the humanoid robot HRP-2.

\section{Movement Database Generation}

The first part of the work was devoted to the synthesis of two databases of reaching movements. The first database was obtained in simulation, by applying the biological model of motor control proposed by Guigon et al. [13], on the dynamical model of HRP-2's arm. The application of this model to the control of HRP-2's reaching movements was described in a previous work [14]. This result is briefly recalled here. Guigon's model is based on the idea that the CNS processes dynamic efforts (inertial, velocity dependent) and static efforts (elastic, gravitational) separately, and that the energy of motoneurons is continuously minimized during movement. In order to apply these principles to the control of HRP-2, a global model was considered, which contained the dynamics of the 6 DOF of the robot arm. For each DOF, an additional filter simulating the dynamics of a pair of virtual antagonist muscles was included. From this model, we determined the reaching trajectories minimizing the energy of the virtual motoneurons, while disregarding the gravitational term in the model, according to the separation principle. The robot arm movements obtained using this approach turns out to be very realistic and exhibit the main kinematic features of human reaching motions. However, the main drawback of this approach is the computation time. Indeed, several minutes were necessary to generate a movement, making the method unusable for on-line control. This motivated our wish to determine a finite set of primitives from which the optimal solutions could be determined faster.

The second database was obtained by recording human movements using a motion capture technology. Arm motion of 3 participants were recorded using infrared markers attached to the shoulder, elbow and wrists. Accuracy of capture was less than $1 \mathrm{~mm}$, with a frequency of $100 \mathrm{~Hz}$. Participants were asked to perform a set of reaching movements while standing up. We considered 32 target positions, regularly-spaced in two parallel grids located in front of the participant's shoulder (Fig. 1). The reaching target was a small ball at the end of a stick that was manipulated by the experimenter. The target was placed at one of the 32 position, and the participants heard a sound to indicate that they should start moving their hand to the target. Upon reaching the current target, the next target was randomly chosen from the other 31 positions, and the experimenter moved the stick to this new position. Movements were executed such that all 32 targets were reached from each other, giving a total of 993 movements per participant.

The same sequence of task was used for simulating the reaching movements with the dynamic model of HRP-2, in order to construct the first database. However, in the case of the robot, a scaling factor was applied to the dimension of the target setup and its distance to the robot in order to obtain comparable values. For both the human subjects and the robot, the database were constructed for a model of arm including six joints: three at the shoulder, two at the elbow and one at the wrist. Note that the remaining DOF of the human arm were not considered for this application. In order to simplify the primitive extraction, the robot movements were all executed with a duration of $1 s$ discretized in 100 time values. In the same way, the human reaching trajectories were normalized to $1 s$ within the same sampling of $0.01 s$. So, for both the robot and the humans, each reaching movement was described by 600 values encoding the variation of 6 angles.

\section{Primitives eXtraction}

We are looking for open-loop primitives with no sensory feedback. In the literature, such primitives are defined as time functions to be modulated in amplitude by weighting coefficients [9], [8], [15]. The most general and simplest way of modeling the problem is to consider that movements can be express as linear combination of these primitives. For our problem, each movement of the 6 DOF arm can be represented by a joint trajectory $u(t) \in \mathbb{R}^{6}$. A database of $M$ movements is then described by a set of such trajectories: $U_{m}(t)=$ $\left(U_{m}^{1}(t), U_{m}^{2}(t), \ldots U_{m}^{6}(t)\right)^{T} \in \mathbb{R}^{6}, m=1, \ldots, M, t \in[0, T]$. For this database, the primitive extraction problem is to determine $K$ time-functions $\Phi_{k}(t)=\left(\Phi_{k}^{1}(t), \Phi_{k}^{2}(t), \ldots \Phi_{k}^{6}(t)\right)^{T} \in$ $\mathbb{R}^{6}, k=1, \ldots, K, t \in[0, T]$, such that, for all $m=1, \ldots, M$, it is possible to determine $k$ real coefficients $\alpha_{k}^{m}$, verifying:

$$
U_{m}(t)=\sum_{k=1}^{K} \alpha_{k}^{m} \Phi_{k}(t)
$$

In this expression, the functions $\Phi_{k}(t)$ represent the expected primitives and the coefficients $\alpha_{k}^{m}$ are weighting the contribution of each primitive $\Phi_{k}(t)$ into the movement $U_{m}(t)$. Each $\Phi_{k}(t)$ has then the same dimension as $U_{m}(t)$, and constitutes a particular joint trajectory. The determination of Eqn. (1) states two problems. The first one is to determine the number, $K$, of primitives that are necessary to reach a given precision and the second one is to characterize these $K$ primitives. To reduce the complexity of the control problem, $K$ needs to be as small as possible, compared to the number of parameters necessary to encode the trajectory $U_{m}(t)$. For a continuous-time problem, this number is infinite. However, in practice, the problem is solved by considering a sampling of the time interval $[0, T]$ in $J$ elements. For the 6 DOF arm we consider, $K$ needs to be small with respect to the $6 \mathrm{~J}$ angle values that encode each movement. In other terms, once the $K$ primitives are determined, it is sufficient to compute $K$ coefficients $\alpha_{k}^{m}, k=1, \ldots, K$, to generate the movement $U_{m}(t)$ over $[0, T]$, instead of $6 J$ variables. The second problem is to compute the canonical time-functions $\Phi_{k}$. Considering the notation of Eqn. (1), each movement $U_{m}$ and each primitive $\Phi_{k}$ is defined by a $6 \times J$ matrix as follows: 


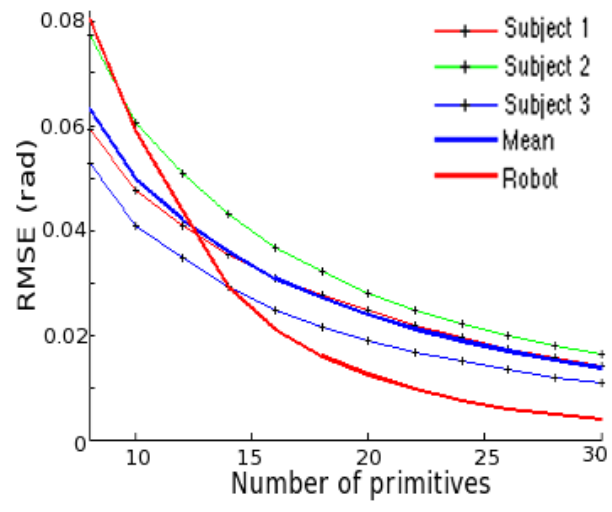

Fig. 2. Root Mean Square Error between original trajectories and reconstructed ones, as a function of the number of primitives $K$. The curves corresponding to the three human subject and the robot arm are represented. In addition, the blue curve represents the mean of the three human subjects.

$$
U_{m}=\left[\begin{array}{cccc}
u_{11}^{m} & u_{12}^{m} & \ldots & u_{1 J}^{m} \\
u_{21}^{m} & u_{22}^{m} & \ldots & u_{2 J}^{m} \\
\ldots & & & \\
u_{61}^{m} & u_{62}^{m} & \ldots & u_{6 J}^{m}
\end{array}\right], \Phi_{k}=\left[\begin{array}{cccc}
\phi_{11}^{k} & \phi_{12}^{k} & \ldots & \phi_{1 J}^{k} \\
\phi_{21}^{k} & \phi_{22}^{k} & \ldots & \phi_{2 J}^{k} \\
\ldots & & & \\
\phi_{61}^{k} & \phi_{62}^{k} & \ldots & \phi_{6 J}^{k}
\end{array}\right]
$$

where, $u_{h j}^{m}$ and, $\phi_{h j}^{k}, h=1, \ldots, 6, m=1, \ldots, M, j=1, \ldots, J$ are real numbers. On this basis, the reconstruction error relative to the $M$ movements of the database, $U_{m}, m=1, \ldots, M$ is defined by:

$$
E=E\left(\alpha_{k}^{m}, \Phi_{k}\right)=\sum_{m=1}^{M} \sum_{h=1}^{6} \sum_{j=1}^{J}\left(u_{h j}^{m}-\sum_{k=1}^{K} \alpha_{k}^{m} \phi_{h j}^{k}\right)^{2}
$$

Solving this problem is then reduced to the determination of $K$ primitives, with $K$ as small as possible, $(K<<6 J)$, but sufficiently large to guarantee that the reconstruction error will be lower than the expected precision threshold. This compromise will be discussed in the next section. Among the existing techniques that allow to cope with this kind of problem (see [18] for an overview), Principal Component Analysis (PCA), is intrinsically well adapted, simple and well-performing. It has already been successfully tested in neurosciences and in robotics [1], [3], [16] [9]. In order to apply PCA, it is more convenient to use a vector notation of data. So, instead of using the matrix notation (Eqn. (2)), each movement $U_{m}, m=1, \ldots, M$ will be described by the following vector expression:

$$
U_{m}=\left(u_{11}, u_{12} \ldots, u_{1 J}, u_{21}, \ldots, u_{2 J}, \ldots, u_{61}, \ldots, u_{6 J}\right)^{T}
$$

where, each $u_{h j}, h=1, \ldots, 6, j=1, \ldots, J$ is the angular value of joint $h$ at time $j$. The $M$ column vectors $U_{m}$ of dimension $N=6 J$ are then gathered to compose a $M \times N$ matrix. The $N \times N$ covariance matrix is then computed. The eigenvectors $e_{i}, i=1, \ldots, N$ and eigenvalues $\lambda_{i}, i=1, \ldots, N$ of this covariance matrix are then determined and ordered. The eigenvalue $\lambda_{i}$ represents the variance of data in the direction of the corresponding eigenvector $e_{i}$. The larger $\lambda_{i}$ is, the more its associated component $e_{i}$ is dominant in the representation of data. The question is then to determine the number $K$, such that the first $K$ principal components $e_{k}, k=1, \ldots, K$ constitute a basis of primitives from which the whole database can be expressed with the required precision. Note that each primitive $e_{k}$ is itself the representation of a six-joints trajectory. Finally, for each movement $U_{m}$, the coefficient corresponding to the primitive $e_{k}$ is determined by computing the scalar product between these two vectors: $\alpha_{k}^{m}=<U_{m}, e_{k}>, m=1 . . M, k=1, \ldots, K$. Note that the application of PCA requires the data to be centered. This condition was almost exactly satisfied for each database.

PCA was separately applied to the robot database and the human databases described in section II. In each case, we observed that the variance accounted for (VAF) by the $K=8$ first primitives was more than $96 \%$, while it was more than $99.5 \%$ with the $K=20$ first primitives. Using the previously described approach, we determined the coefficients that allowed us to reconstruct the whole set of movements by a linear combination of these primitives. These reconstructed trajectories were then compared with the original ones. To compute the reconstruction error for a whole database, we used the Root Mean Squared Error (RMSE), which is expressed as follows:

$$
R M S E=\sqrt{\frac{E}{(M \times 6 \times J)}}
$$

where, $E$ is defined by Eqn. (3). Fig. 2 shows the decay of the RMSE as a function of the number $K$ of primitives, for each database. The decay rate of the RMSE is similar for the three human subjects, while it is slightly different for the robot arm. For human subjects, beyond the first 8 primitives, each addition of a new primitive induces almost the same error reduction. Whereas, for the robot model, the important decay rate between the $8^{\text {th }}$ and the $16^{\text {th }}$ primitive shows that these primitives still contain an important part of information. Interestingly, the curves corresponding to the human subjects and the robot become roughly parallel after the $18^{\text {th }}$ primitive, showing a certain level of similarity between both databases. Beyond this level, the regular gap between the curves seems to be due to the difference in the kinematics and the dynamics of the arm structures. As human subject are taller and heavier than the robot, the effect of inertia and masses are higher on the human arm than on the robot arm. This might induce a higher reconstruction error for human movements. In each case, the primitives computed with PCA allow to represent the original data with a good precision. Depending on the expected level of precision, the number of primitives can be easily chosen. For instance, $K=16$ primitives allows to reproduce the robot database with a mean error of 0.02 radians, $\left(1.2^{\circ}\right)$. The same number of primitives allows to represent the database of subject 1 with a mean error of 0.03 radians, $\left(1.7^{\circ}\right)$. As an example, Fig. 3 shows two trajectories reconstructed from these primitives and Fig. 4 shows the first 8 primitives for each database: the robot, subject 1,2 and 3. The primitives represent the principal variations of the movements, classified by decreasing order of dominance variance. The first 


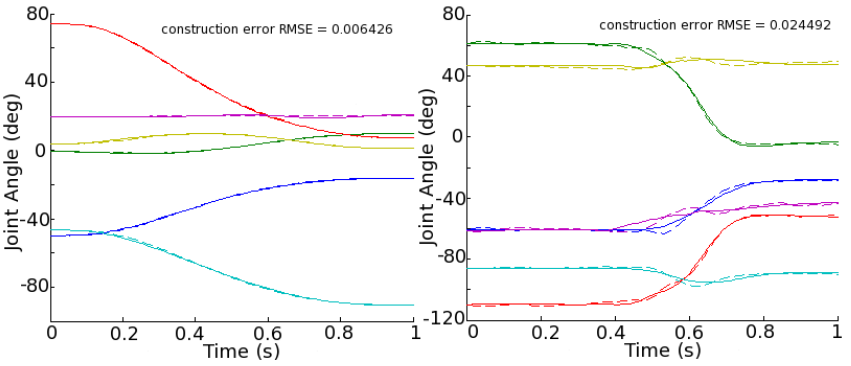

Fig. 3. Example of joint trajectories reconstructed from $K=16$ primitives. The curves on the left picture were obtained with the robot database, whereas the curves on the right picture were obtained with the database of subject 1 . The original trajectories are in dotted lines and the reconstructed trajectories in full line.

primitives are rather regular, while the last more oscillatory one, allows to capture subtle variations of movements. Recall that each primitive describes the 6 joint trajectories. It is interesting to remark in Fig. 4, that the curves of primitives corresponding to the different database are not necessarily similar. This difference is due to the definition of primitives given by (1). Indeed, according to this relation, each primitive represents the simultaneous temporal evolution of the six arm joints, and these six trajectories are weighted by the same coefficient $\alpha_{k}^{m}$. This choice is biologically plausible, but leads to a different primitive basis of each database. Indeed, even though the subjects do the reaching movements in a similar way, the variations due to each subject may radically modify the expression of primitives.

It is important to note that some authors used PCA to extract primitive for each link separately [9]. As a consequence, the modulation of primitives is decoupled on each joint. In that case, it was shown that a lower number $K$ of primitives is sufficient for each link (usually between 2 and 4), but the number of coefficients is multiplied by $N$. Thus, if 3 primitives are necessary for each link of a 6 DOF arm, then 18 primitives will be necessary for representing the arm movements. We also tested this approach for our problem and we found that the variance accounted for by 3 primitives on each link was more than $96 \%$. In particular, we found a certain similarity between the primitives for the subject and the robot arm. However, there is no fundamental difference between the two methods of primitive extraction. The number of variables are almost identical in both cases. By looking for primitives containing the 6 arm joints, our goal was to capture the joints co-activation. In this way, we expected to reduce the complexity, increase the precision of reconstructed movements, and find a basis of primitives well adapted to generalization. This last step is considered in the next section.

\section{Generalization}

So far, we have shown that large databases of reaching movements can be expressed as linear combination of a small number of primitives. The question is now to determine if it is possible, from these primitives, to generate new movements having the characteristics of human movements. In other terms, we want to solve a generalization problem

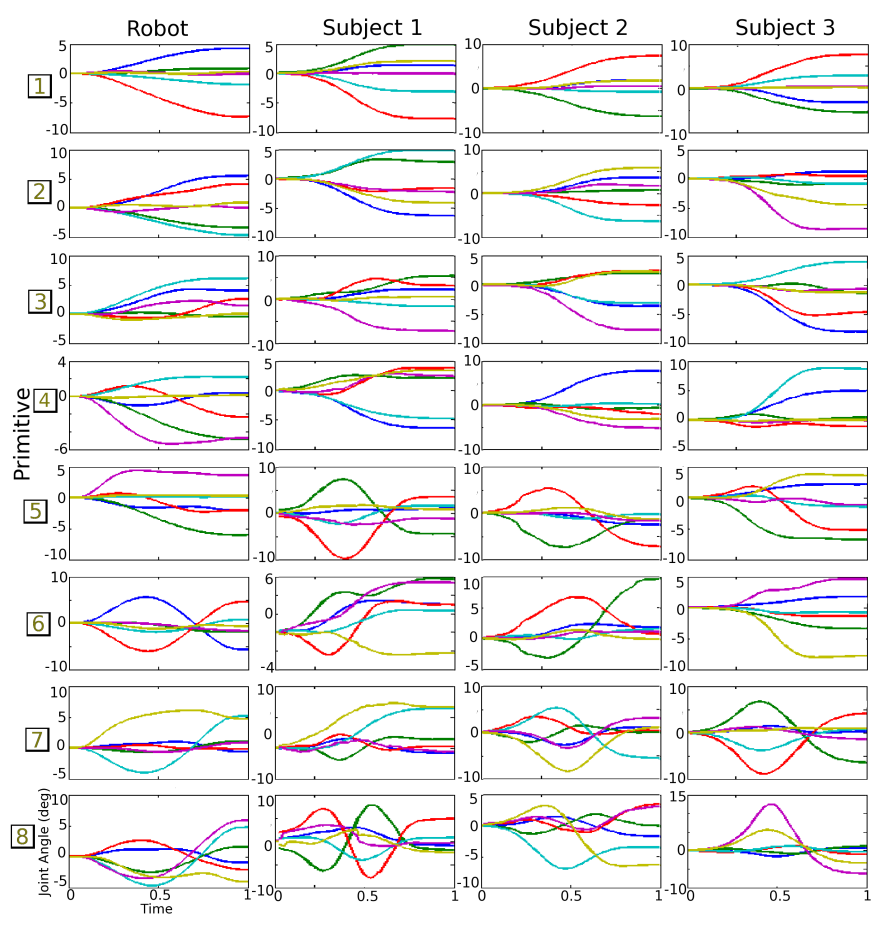

Fig. 4. Description of the first 8 primitives obtained with PCA on the different databases. From left to right the column correspond to the robot, subject 1, 2 and 3 respectively. From top to down, primitives are classified by variance dominance. In each scheme, the abscissa axis represents the time in seconds and the ordinates represent the joint angles in degrees. A different color is used for each of the 6 arm joints

which can be stated as follows: considering a set of primitives $\Phi_{k}$, given the initial arm configuration and a reaching task defined by the the target position and the movement duration, determine the coefficients $\alpha_{k}$ that allow to express the trajectory as a linear combination of the primitives.

However, recall that the objective of this work is to generate realistic reaching movements rapidly, without having to solve a complex optimization problem as we did previously in [14]. Our first attempt to solve the generalization problem was to apply learning methods in order to characterize fast input-output interface. We tested different architectures of multi-layer feedforward neural networks which received as input the six values of initial arm joints, the three Cartesian coordinates of the target and the movement duration, and were expected to give the $\alpha_{k}$ weighting coefficients of the $K$ primitives as output. For some of these networks, the learning process on the 993 input-output pairs of each database took several hours. However, none of them succeeded in characterizing the $\alpha_{k}$ in a sufficiently generic way. In many cases, the reaching error between the final hand position and the target was too large and the movements were often not realistic. Increasing the number of primitives $K$ did not improve the quality of the result. It seemed that the information learned by the networks was not sufficiently rich to capture the movement characteristics. Furthermore, feedforward neural network does not seem to be well appropriate to cope with the sensibility of the weighting of motor primitives. Then, as the learning techniques we considered did not provide us 


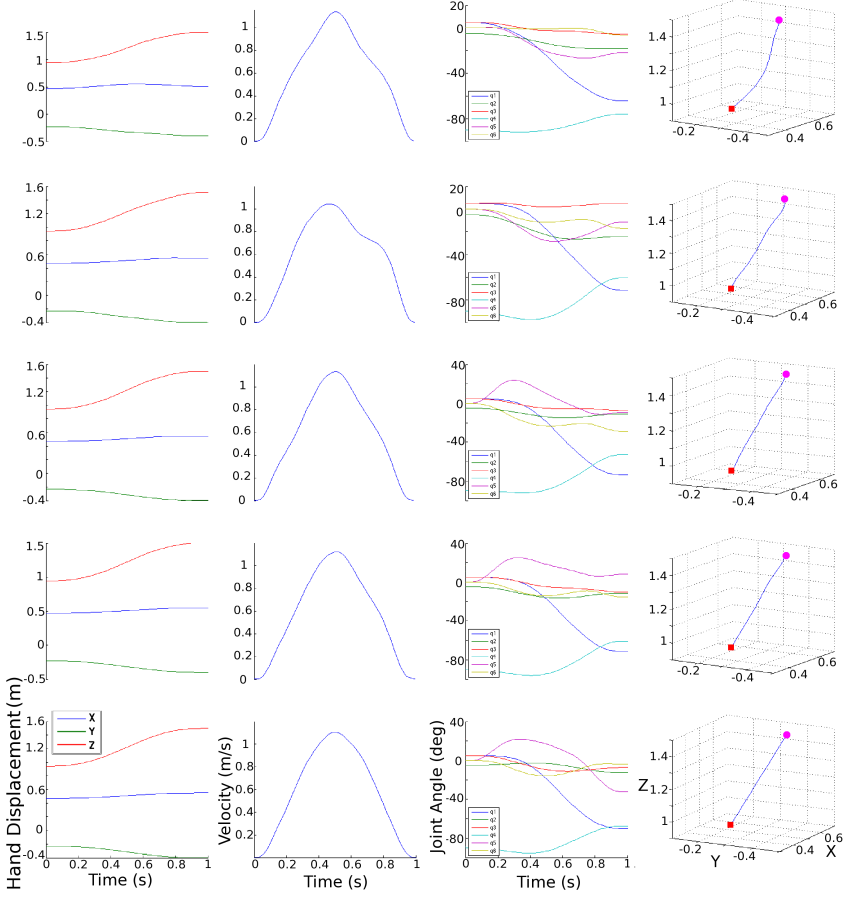

Fig. 5. For a same reaching task of amplitude $58 \mathrm{~cm}$, description of the movement successively generated by using an increasing number primitives $K=6,8,10,12$, and 20 . From left to right, each horizontal set of 4 pictures corresponds to a particular value of $K$ and represents: the Cartesian hand coordinates, the hand velocity profile, the joint trajectories and the hand trajectory. The reaching errors in $\mathrm{cm}$ are respectively: $4.07,1.14,0.36,0.12$ and 0.11 .

with satisfying results, we developed an original approach which consists in constraining the hand trajectory in the operational space. This method is described in the following section.

\section{A. Method of trajectory constraint in the operational space}

We want to determine the $\alpha_{k}$ that allow to generate new movements that still have the characteristic of human movements. To this end, we propose to determine the $\alpha_{k}$ which guarantee that the hand trajectory satisfies the minimum jerk condition [17]. In order to implement this reasoning, we formulate an optimization problem whose objective is to characterize the $\alpha_{k}$ coefficients that allow to minimize the mean error between the hand trajectory and the minimumjerk curve in the Cartesian space. As before, the time interval $[0, T]$ is sampled in $J$ elements $0=t_{1}, . ., t_{J}=T$. For $j=1, \ldots, J$, let us denote by $q_{j}=\left(q_{1 j}, q_{2 j}, q_{3 j}, q_{4 j}, q_{5 j}, q_{6 j}\right)^{T}$ the arm configuration at time $t_{j}$ and $H_{j}=f\left(q_{j}\right)$ the corresponding hand position in the Cartesian space, where $f$ represents the direct kinematics associated to the $6 \mathrm{DOF}$ arm model. Using this notation, the optimization problem can be stated as follows: Given $K$ primitives $\Phi_{k}, k=1 . . K$, each one representing the six arm joint trajectories, the initial arm configuration $q_{1}$, and a reaching task defined by the target position $H_{f}$, find the value of $K$ real coefficients $\alpha_{k}, k=1, \ldots, K$, minimizing the function $E_{\text {jerk }}$ defined by:

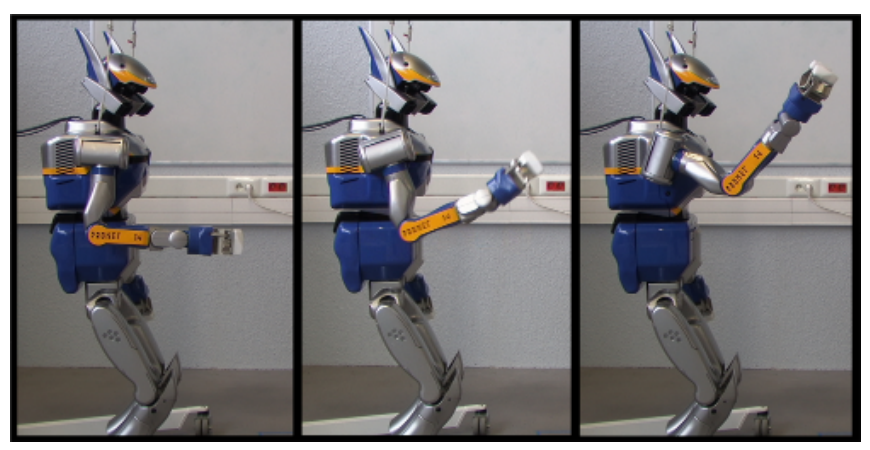

Fig. 6. Snapshots of HRP-2 during the execution of reaching motion. The motion here corresponds to the curves of Fig. 5 for $K=20$ primitives.

$$
E_{j e r k}=\sum_{j=1}^{J}\left\|f\left(q_{j}\right)-g\left(\tau_{j}, H_{1}, H_{f}\right)\right\|
$$

Recall that, according to the definition of primitives, the angular value $q_{h j}$ of joint $h$ at time $t_{j}$, that appear in the above definition, is computed from the relation $q_{h j}=q_{h 1}+$ $\sum_{k=1}^{K} \alpha_{k} \phi_{h j}^{k}$, where $\phi_{h j}^{k}, j=1, \ldots, J$, is the angular value of the $h^{\text {th }}$ joint of the $k^{\text {th }}$ primitive at time $t_{j}$, and $g$ is the timefunction representing the hand position along the reference minimum-jerk trajectory expressed by:

$$
g(\tau)=H_{1}+\left(H_{1}-H_{f}\right)\left(15 \tau^{4}-6 \tau^{5}-10 \tau^{3}\right),
$$

where $\tau$ is the normalized time and $\tau_{j}=t_{j} / T$ the discrete normalized value that corresponds to time $t_{j}$ in $[0, T]$. In this problem, the joint limits on the $q_{h j}$ during the movement are also considered. They are defined by an inequality of the type:

$$
l_{h} \leq q_{h j} \leq u_{h} \quad \text { for } h=1, \ldots, 6, j=1, \ldots, J,
$$

where $l_{h}$ and $u_{h}$ are the upper and lower bounds of joint $h$ respectively. Note that this problem is different from the one considered in [9]. Here, the objective is not to minimize a kinematic or dynamic criterion to determine the control signals, but instead to follow a reference curve, having the characteristics of human movement, in the Cartesian space. This problem is also different from the usual minimum-jerk problem in the sense that the joint trajectory of the arm is directly deduced from the knowledge of the $\alpha_{k}$ coefficients. No additional computation is then required to compute the joint trajectories; a step which usually requires to solve the inverse kinematics problem with the minimization of some additional criterion to cope with redundancy. Furthermore, note that the generalization problem described by Eqn. (5), (6) and (7) is a simple optimization problem involving $K$ real variables and $6 \mathrm{~J}$ linear constraints, which can be solved by using standard techniques. For this work, we used the Matlab fmincon solver. The result we obtained is described in the next section.

\section{B. Results}

In order to test the proposed generalization approach, a large number of new movements were generated by considering the model of the robot arm. To this end, a shift 
TABLE I

MEAN ERROR AND COMPUTATION TIME FOR DIFFERENT $K$

\begin{tabular}{|c|c|c|}
\hline \hline $\mathrm{K}$ & Mean error $(\mathrm{cm})$ & Computation time $(\mathrm{s})$ \\
\hline 6 & 3.6504 & 5.5549 \\
8 & 1.6120 & 11.1249 \\
10 & 1.3822 & 14.1024 \\
12 & 1.1417 & 20.5878 \\
14 & 1.1169 & 28.3012 \\
16 & 1.0842 & 35.5586 \\
18 & 1.0415 & 41.1163 \\
20 & 0.9676 & 48.6022 \\
\hline
\end{tabular}

between $-10 \mathrm{~cm}$ and $+10 \mathrm{~cm}$ was applied at random in each direction of the initial target grid (see Fig. 1) to generate new targets, and angular increments between $-5^{\circ}$ and $+5^{\circ}$ were randomly added to each arm joint to specify new initial arm configurations. This allowed to generate a new large set of reaching tasks with different arm configurations. Note that the larger amplitude movements were about 1 meter long. In order to evaluate the compromise between rapidity and reaching precision, different values of $K$ between 6 and 20 were considered. Table I shows the average reaching error and computation time on all tested movements for each value of $K$. Logically, for a higher $K$ the computation time increases, while the reaching error decreases. It can be noted that the reaching error defined by the distance between the target position and the hand position at final instant $T$ can reflect the error at each time instant on the whole trajectory because the term $E_{\text {jerk }}$ in definition (5) is the sum of all instantaneous errors. Fig.5 shows the characteristics of movements obtained with the robot arm, for $K=6,8,10,12,20$. Clearly, the hand trajectories and the velocity curves are not realistic for $K=6$ and 8. However, as $K$ increases, the trajectory becomes straighter and the velocity curve becomes smoother and more regular. For $K=12$ the movement starts being very realistic. The improvement obtained by adding a new primitive is very small. For $K=20$ the velocity profiles are perfectly bellshaped and the hand trajectories are almost straight with a continuous weak curvature. Fig. 6 shows HRP-2 performing the motion corresponding to $K=20$. For the sake of safety, we slowed down the motion to $2 \mathrm{~s}$ instead of the original 1 s. The human and humanoid experimental trials can be seen in the video submitted with this paper.

Compared to our preceding result, where reaching trajectories were obtained by solving a complex optimization problem defined by Guigon's model [14], the computation time reduced by a factor of 6 , i.e. from $2 \mathrm{~min}$ to $20 \mathrm{~s}$. However it is important to note that the method proposed in [14], was encoded in $C$ whereas, so far, the current method is implemented in Matlab. Therefore, we can claim that the computation time can be further reduced by at least a factor 5 , by using the $C$ programming language for encoding the current method. In this condition, less that $5 s$ would be necessary to generate a new realistic reaching movement

\section{CONCLUSION}

This result shows that the use of movement primitives coupled with an appropriate generalization process provides an efficient way to store the complex information of human movement, in order to quickly generate new trajectories. Although it has been suggested that the CNS plans movements by solving complex optimization problems - such as minimizing the energy of motoneurons - it is likely that the use of a reduced number of canonical variables allows to simplify the computation. The application of this kind of approach to humanoid robotics is interesting as it offers an alternative to the computation of inverse kinematics with additional cost minimization. In future works, we plan to address the problem of combining such open-loop primitives to produce whole body movements.

\section{ACKNOWLEGMENTS}

This work was supported by the Zeuxis project funded by the EADS foundation and by the ROMEO project funded by the French FUI.

\section{REFERENCES}

[1] A. Alexandrov, A. Frolov and J. Massion, Axial synergies during human upper trunk bending, Exp Brain Res, 118(2), 1998, pp210220.

[2] F. Lacquaniti, R. Grasso, and M. Zago, Motor patterns in walking, News Physiol Sci, 14, 1999, pp 168-174.

[3] J.S. Thomas, D.M. Corcos, and Z. Hasan, Kinematic and kinetic constraints on arm, trunk, and leg segments in target-reaching movements, J Neurophysiol, 93(1), 2005, pp 352-364.

[4] T.R. Kaminski, The coupling between upper and lower extremity synergies during whole body reaching, Gait Posture, 26(2), 2007 pp256-262.

[5] G. Torres-Oviedo, J.M. Macpherson, and L.H. Ting, Muscle synergy organization is robust across a variety of postural perturbations. $J$ Neurophysiol, 96(3), 2006, pp 1530-1546.

[6] F.A. Mussa-Ivaldi and E. Bizzi, Motor learning through the combination of primitives, Phil. Trans. R. Soc. Lond. B 355, 2000, pp 17551769.

[7] F. Nori, and R. Frezza, A control theory approach to the analysis and synthesis of the experimentally observed motion primitives. Biol. Cybern. 93(5), 2005, pp 323-342.

[8] H. Hauser, G. Neumann, A.J. Ijspeert, and W. Maass, Biologically inspired kinematic synergies provide a new paradigm for balance control of humanoid robots, Int. Conf. Proc. IEEE-RAS Humanoids, 2007.

[9] B. Lim, S. Ra, and F.C. Park, Movement primitives, principal component analysis, and the efficient generation of natural motions, IEEE Int. Conf. ICRA 2005.

[10] M. Chhabra, and R. Jacobs, Properties of synergies arising from a theory of optimal motor behavior, Neural Computation, 18(10), 2006, pp 2320-2342.

[11] B. Berret, F. Bonnetblanc, C. Papaxanthis, and T. Pozzo, Modular control of pointing beyond arm's length, Journal of Neuroscience, 29(1) 2009.

[12] E. Todorov and Z. Ghahramani, Unsupervised learning of sensorymotor primitives, IEEE Int. Conf. EMBS, 2003.

[13] E. Guigon, P. Baraduc, and M. Desmurget, Computational motor control: Redundancy and invariance. J. of Neurophysiol 97(1), 2007, pp 331-347.

[14] M. T. Tuan, P. Souères, M. Taïx, E. Guigon, ”A principled approach to motor control for generating humanoid robot reaching movements, IEEE International conference on Biomedical Robotics and Biomechatronics, Scottsdale, Arizona, USA, Oct. 2008.

[15] M. Tresch, V. Cheung, and A. d'Avilla, Matrix factorization algorithms for the identification of muscle synergies: evaluation on simulated and experimental data sets, J. of Neurophysiol., 95, 2006, pp 2199-2212.

[16] T.D. Sanger, Human arm movements described by a low-dimensional superposition of principal components, J. Neurosci. 20, 2000, pp 10661072.

[17] T. Flash and B. Hochner, Motor primitives in vertebrates and invertebrates, Current Opinion in Neurobiology 15 (6) 2005, pp 660-666.

[18] I.K. Fodor, A survey of dimension reduction techniques, LLNL Technical Report, UCRL-ID-148494, 2002. 